\title{
Oropharyngeal Dysphagia
}

National Cancer Institute

\section{Source}

National Cancer Institute. Oropharyngeal Dysphagia. NCI Thesaurus. Code C113588.

Difficulty in swallowing due to an abnormality in the mouth or throat. 